\title{
FLOATING-HARBOR SYNDROME: PRESENTATION OF THE FIRST ROMANIAN PATIENT WITH A SRCAP MUTATION AND REVIEW OF THE LITERATURE
}

\author{
Budisteanu $\mathrm{M}^{1,2}$, Bögershausen $\mathrm{N}^{3}$, Papuc $\mathrm{SM}^{2}$, Moosa $\mathrm{S}^{3}$, Thoenes $\mathrm{M}^{4}$, Riga $\mathrm{D}^{1}$, Arghir A $\mathrm{A}^{2,}$, Wollnik B ${ }^{3}$
}

\begin{abstract}
*Corresponding Author: Aurora Arghir, M.D., Ph.D., Victor Babes National Institute of Pathology, Medical Genetics Laboratory, 99-101 Splaiul Independentei, 050096 Bucharest, Romania. Tel/Fax: +40-21-319-27-32, ext. 207/+40-21-319-45-28. E-mail: aurora.arghir@ivb.ro
\end{abstract}

\begin{abstract}
Floating-Harbor syndrome (FHS) is a rare autosomal dominant syndrome characterized by short stature with delayed bone age, retarded speech development, intellectual disability and dysmorphic facial features. Recently, dominant mutations almost exclusively clustered in the final exon of the Snf2-related CREBBP activator protein (SRCAP) gene were identified to cause FHS. Here, we report a boy with short stature, speech delay, mild intellectual disability, dysmorphic features, and with genetically confirmed FHS. To the best of our knowledge, this is the first molecularly confirmed case with this syndrome reported in Romania. An intensive program of cognitive and speech stimulation, as well as yearly neurological, psychological, ophthalmological, otorhinolaryngological, pediatric and endocrinological monitoring for our patient were designed. We propose a checklist of clinical features suggestive of FHS, based on the main clinical features, in order to facilitate the diagnosis and clinical management of this rare condition.
\end{abstract}

Keywords: Clinical management; Floating-Harbor syndrome (FHS); Snf2-related CREBBP activator protein $(S R C A P)$ gene.

\section{INTRODUCTION}

Floating-Harbor syndrome (FHS, MIM 136140) is a rare genetic condition characterized by proportionate short stature, delayed bone age, expressive language delay

\footnotetext{
${ }^{1}$ Professor Dr. Alex Obregia Clinical Hospital of Psychiatry, Bucharest, Romania

${ }^{2}$ Victor Babes National Institute of Pathology, Bucharest, Romania

${ }^{3}$ Institute of Human Genetics, University Medical Center Göttingen, Göttingen, Germany

${ }^{4}$ Institute of Human Genetics, University of Köln, Köln, Germany
}

and distinctive facial features [1]. The syndrome was first described in two patients at the Boston Floating Hospital [2] and at the Harbor General Hospital in Torrance, CA, USA, respectively, hence the name [3]. Its prevalence is unknown; if we take into account only SRCAP mutated cases, around 65 individuals have been reported to date.

The FHS is caused by heterozygous mutations in exons 34 [4-6] and 33 [7,8] of the SRCAP gene, on chromosome 16p11.2. The $S R C A P$ gene mutations are de novo truncating mutations. The SRCAP protein activates the $C R E B B P$ gene that is involved in the regulation of cell growth and division.

Growth deficiency in FHS becomes apparent in the first year of life, but it can occur before birth. Affected children have a short stature with an average height below the 5 th percentile. Bone age is delayed in the first decade of life.

Typical facial features in patients with FHS include a triangularly-shaped face, low-set ears, low hairline, deep-set eyes with abnormally long eyelashes, a long, triangular-shaped nose with a low hanging columella, a short philtrum, and a broad, linear mouth with thin lips [9]. Expressive language delay is a common feature, varying from mild to very severe. Most affected children show some degree of intellectual disability, ranging from mild to severe; some patients have behavioral troubles (hyperactivity, attention deficit, aggression, obsessive behavior). Patients with FHS tend to have an unusually high-pitched voice. Other symptoms reported in individuals with FHS include skeletal anomalies (brachydactyly, fifth finger clinodactyly, 11 pairs of ribs, kyphoscoliosis), congenital heart malformations (aortic coarctation, atrial septal defect, tetralogy of Fallot), gastrointestinal features (motility problems, celiac disease), genitourinary abnormalities (kidney agenesis, renal cysts, hydronephrosis, precocious puberty, cryptorchidism, hypospadias), dental anomalies (supernumerary teeth, microdontia, malocclu- 
sion, delayed loss of primary teeth), ear anomalies (recurrent otitis media, conductive hearing loss), ophthalmologic issues (hyperopia, refractive errors, strabismus), seizures and hypothyroidism [9].

Here, we report on a patient with growth deficiency, dysmorphic facial features, language delay, and mild intellectual disability, with a known pathogenic mutation c. $7330 \mathrm{C}>\mathrm{T}$, p. $\left(\operatorname{Arg} 2444^{*}\right)$ in the SRCAP gene. To the best of our knowledge, this is the first case of molecularly proven FHS in Romania.

Clinical Report. A 7-year-old Romanian boy was referred to the Pediatric Neurology Department, for speech delay. The boy is the second child of healthy, unrelated parents. The pregnancy was uncomplicated, fetal ultrasound revealed short humerus (at 5 months of gestation). The child was born at 40 weeks with a birth weight of $2800 \mathrm{~g}, 51 \mathrm{~cm}$ in length, and good postnatal adaptation. The child presented normal motor development, but delayed language development (he uttered first syllables at 27 months and first words at 4 years 6 months. Hypospadias was diagnosed at birth and surgically corrected at 1 year; the boy had frequent respiratory and middle ear infections. In the first year of life, he presented feeding difficulties and failure to thrive.

Clinical examination at 7 years old revealed: a height of $107 \mathrm{~cm} \mathrm{[<2} \mathrm{standard} \mathrm{deviation} \mathrm{(SD),} \mathrm{a} \mathrm{weight} \mathrm{of} 17 \mathrm{~kg}$ $(<2 \mathrm{SD})$, an occipito-frontal circumference (OFC) of 50 cm (10th percentile). He had dysmorphic facial features: a triangularly-shaped face, deep-set eyes, long eyelashes, lowset malformed ears (hypoplastic helix, small ear lobe), a long nose with narrow bridge, a short philtrum, thin lips, microdontia, malocclusion, and a low frontal hairline (Figure 1).

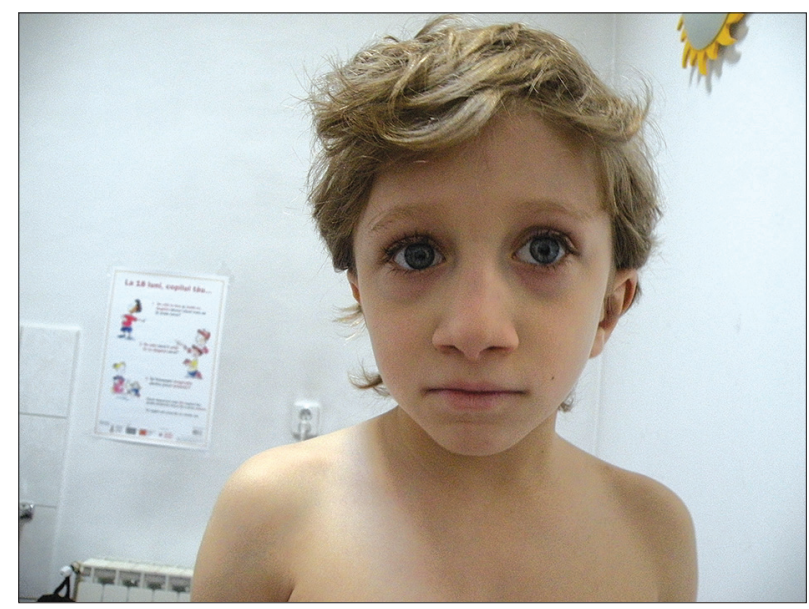

Figure 1. Frontal view of the patient's face showing dysmorphic facial features: a triangularly-shaped face, deep-set eyes, long eyelashes, low-set malformed ears, a long nose with narrow bridge, a short philtrum, thin lips.
He also had mild mental retardation (IQ 66), severe expressive language delay (few simple sentences), hyperkinesia and attention deficit, episodes of aggressiveness at minor frustrations. Skeletal anomalies (brachydactyly, broad finger tips) were noted at clinical investigation. Xray examination showed a bone age of 3 years at the age of 6. Cerebral magnetic resonance imaging (MRI) was normal, including normal pituitary anatomy, as were heart and abdominal ultrasound. Ophthalmologic evaluation was also normal. The levels of thyroid and growth hormones were within the normal range. Taking into account the association of growth deficiency with delayed bone age, expressive language delay and distinctive facial features, a clinical diagnosis of FHS was established. Written consent for publishing clinical data and images of the patient was obtained from the parents.

Sanger sequencing of exons 33 and 34 of the $S R$ $C A P$ gene identified the heterozygous mutation c. $7330 \mathrm{C}>\mathrm{T}$ on exon 34. At the protein level, this sequence alteration leads to truncation of the protein at position 2444 (p.Arg2444*). This mutation is reported in the databases dbSNP (rs 199469464; http:/www.ncbi.nlm.nih.gov/ SNP/), Clin Var (RCV000023895; http://www.ncbi.nlm. nih.gov/clin var/) and HGMD (https://portal.biobaseinternational.com/ hgmd/pro/start.php) as a pathogenic variant. Segregation analysis revealed that both parents do not carry the mutation c. $7330 \mathrm{C}>\mathrm{T}$, showing that it has occurred de novo in the patient.

According to current recommendations, we instituted an intensive program of cognitive and speech stimulation, using picture exchange communication system (PECS), and behavioral therapy. Yearly neurological, psychological, ophthalmological, otorhinolaryngological, pediatric and endocrinological, monitoring of our patient was planned.

\section{DISCUSSION}

Hood et al. [4] first demonstrated mutations in the SRCAP gene to be the cause of FHS in 2012. They reported a series of 13 patients who all carried truncating mutations within a small region of exon 34 (codons 2407 to 2517). Mutations could be proven to be de novo for all patients for whom parental DNA was available. In 2013, Le Goff et al. [5] published a cohort of nine patients. Six of them presented mutations of the SRCAP gene. The biggest cohort of patients with FHS yet reported was published by Nikkel et al. [6], who described 52 patients with mutations on the $S R C A P$ gene, including the 13 patients previously reported by Hood et al. [4]. Nikkel et al. [6] refined the clinical spectrum of FHS and demonstrated autosomal-dominant in- 
heritance in two mother/daughter pairs. They also extended the critical molecular region to between codons 2389 and 2748 [6]. The most recent series of five patients with FHS and mutations in the SRCAP gene was published in 2014 by Seifert et al. [8], who confirmed that exon 33 of the $S R C A P$ gene can also be affected by pathogenic mutations, as previously reported by Kehrer et al. [7].

The main clinical features of FHS are the characteristic facial features (triangular face, long nose with narrow root and broad tip, low hanging columella, large nares, short philtrum, thin vermillion border of the lips, everted lower lip, a linear orientation of the mouth at rest or when smiling, deep set eyes, long eyelashes, low set, large ears), growth deficiency, language delay, skeletal anomalies (including brachydactyly, broad fingertips, short broad thumbs, big toes, clavicular anomalies, hip dysplasia), and delayed bone age. Intellectual disability was reported in many, but not in all patients, varying from mild to severe. Nikkel et al. [6] pointed out that language delay and sensory impairments might confound cognitive assessment in children with FHS. However, most patients did have schooling difficulties. Behavioral problems were noted in two studies (Nikkel et al. [6], Seifert et al. [8]) and included aggressive behavior, anxiety, rigid mannerisms, obsessions, and attention deficit hyperactivity disorder (ADHD). Other features, such as cardiac, gastrointestinal and genitourinary malformations, dental issues, eye and ear anomalies, seizures and hypothyroidism, were reported less frequently. Our patient presented all of the main clinical features in addition to mild intellectual disability, behavioral problems (ADHD), dental anomalies, and genital malformation. We summarize the published clinical data in comparison to the patient presented here in Table 1.

Genetic testing of our patient detected the heterozygous mutation c.7330C $>\mathrm{T}$ in exon 34 of the SRCAP gene, which is a recurrent mutation in FHS patients; Hood et al. [4] identified the c.7330 $>\mathrm{T}$ mutation in six out of 13 patients and Nikkel et al. [6] found this mutation in 24/52 patients. Nikkel et al. [6] proposed that mutations in the last exons of the $S R C A P$ gene should be considered mandatory for the diagnosis of FHS, in the presence of the classic phenotype. Thus, the patient presented here conforms to all of the published diagnostic criteria for FHS.

A straightforward approach to clinical diagnosis of FHS is essential, especially in countries with limited resources. Therefore, we propose a checklist of clinical

Table 1. Comparison of clinical features reported in the literature (cases with a SRCAP gene mutation) and in our present patient.

\begin{tabular}{|l|c|c|c|c|c|}
\hline Features & \multicolumn{4}{|c|}{ Literature } & This Study \\
\hline References & {$[4]$} & {$[5]$} & {$[6]$} & {$[8]$} & \\
\hline Facial gestalt & $13 / 13$ & $6 / 6$ & $52 / 52$ & $5 / 5$ & {$[+]$} \\
\hline Language delay & $13 / 13$ & $6 / 6$ & $52 / 52$ & $5 / 5$ & {$[+]$} \\
\hline Growth deficiency & $13 / 13$ & $6 / 6$ & $39 / 52$ & $5 / 5$ & {$[+]$} \\
\hline Delayed bone age & $13 / 13$ & $6 / 6$ & $23 / 25$ & $4 / 5^{\mathrm{a}}$ & {$[+]$} \\
\hline Skeletal anomalies & $10 / 13$ & $6 / 6$ & {$[+]^{\mathrm{b}}$} & $5 / 5$ & {$[+]$} \\
\hline Intellectual disability & $6 / 13$ & $3 / 6$ & $37 / 43^{\mathrm{c}}$ & $4 / 5$ & {$[+]$} \\
\hline Behavioral problems & $\mathrm{NR}$ & $\mathrm{NR}$ & {$[+]^{\mathrm{d}}$} & $3 / 5$ & {$[+]$} \\
\hline Eye anomalies & $4 / 13$ & $2 / 6$ & $13 / 43$ & $0 / 5$ & {$[-]$} \\
\hline Ear anomalies & $4 / 13$ & $0 / 6$ & $15 / 52$ & $2 / 5$ & {$[-]$} \\
\hline Dental issues & $4 / 13^{\mathrm{e}}$ & $\mathrm{NR}$ & $21 / 38$ & $1 / 5$ & {$[+]$} \\
\hline Genitourinary malformations & $8 / 13$ & $1 / 6$ & $5 / 24$ & $0 / 5$ & {$[+]$} \\
\hline Cardiac malformations & $3 / 13$ & $3 / 6$ & $3 / 52$ & $0 / 5$ & {$[-]$} \\
\hline Gastrointestinal malformations & $2 / 13$ & $0 / 6$ & $5 / 24$ & $0 / 5$ & {$[-]$} \\
\hline Seizures & $0 / 13$ & $1 / 6$ & $6 / 52$ & $0 / 5$ & {$[-]$} \\
\hline Hypothyroidism & $\mathrm{NR}$ & $0 / 6$ & $2 / 52$ & $0 / 5$ & {$[-]$} \\
\hline
\end{tabular}

NR: not reported; ADHD: attention deficit hyperactivity disorder.

a The X-ray for bone age was not available in one case.

b The authors report on different skeletal anomalies: broad thumb in 10/17 patients, broad toes, brachydactyly, broad fingertips (described as frequent), clavicular anomalies (six patients), hip dysplasia (four cases).

c Patients with learning difficulties.

d The authors report obsessive tendencies, rigid mannerisms (7/25) and ADHD (9/32).

e Not reported in eight cases. 
Table 2. Checklist of Floating-Harbor syndrome clinical features to facilitate clinical diagnosis and management.

\begin{tabular}{|c|c|}
\hline Features & Category \\
\hline Characteristic face & mandatory $^{\mathrm{a}}$ \\
\hline Language delay & mandatory \\
\hline Short stature & frequent $^{\mathrm{a}}$ \\
\hline Delayed bone age & frequent \\
\hline Skeletal anomalies & frequent \\
\hline Intellectual disability & frequent \\
\hline Behavioral problems & recurrent $t^{b}$ \\
\hline Eye anomalies & recurrent \\
\hline Ear anomalies & recurrent \\
\hline Dental issues & recurrent \\
\hline Genitourinary malformations & infrequent $^{\mathrm{b}}$ \\
\hline Cardiac malformations & infrequent \\
\hline Gastrointestinal features & infrequent \\
\hline Seizures & infrequent \\
\hline Hypothyroidism & infrequent \\
\hline
\end{tabular}

FHS: Floating-Harbor syndrome.

a Diagnosis of FHS requires meeting both mandatory criteria and at least one criterion from the frequent features category.

${ }^{\mathrm{b}}$ Recurrent and infrequent categories include features that are not required for diagnosis, but further support FHS diagnosis and guide the clinical evaluation, planning and patient care, if present.

features suggestive of FHS (Table 2), based on the main clinical features defined by Nikkel et al. [6], to facilitate decision making with regard to molecular genetic testing and clinical evaluation planning.

Floating-Harbor syndrome mandatory features are the characteristic facial gestalt and language delay, present in all patients reported and in the patient presented here. Growth retardation, skeletal anomalies and delayed bone age, as well as intellectual disability of varying degrees and behavioral problems, are quite frequently observed in FHS and we believe that at least one of these features should be present in addition to the mandatory features, as proposed in the checklist (Table 2). The other clinical findings (in the recurrent or infrequent categories in our checklist) are not required for a FHS diagnosis; however, their presence increase the confidence of FHS diagnosis and guide the clinical management.

Declaration of Interest. The authors report no conflicts of interest. The authors alone are responsible for the content and writing of this article.

\section{REFERENCES}

1. Robinson PL, Shohat M, Winter RM, Conte WJ, Gordon-Nesbitt D, Feingold M, et al. A unique association of short stature, dysmorphic features, and speech impairment (Floating-Harbor syndrome) J Pediatr. 1988; 113(4): 703-706.

2. Pelletier G, Feingold M. Case report 1. In: Bergsma D, Eds. Syndrome Identification. White Plains (NY, USA): National Foundation-March of Dimes. 1973;1(1):8-9.

3. Leisti J, Hollister DW, Rimoin DL. The FloatingHarbor syndrome. Birth Defects Orig Artic Ser. 1975; 11(5): 305.

4. Hood RL, Lines MA, Nikkel SM, Schwartzentruber $\mathrm{J}$, Beaulieu C, Nowaczyk MJ, et al. Mutations in $S R C A P$, encoding SNF2-related CREBBP activator protein, cause Floating-Harbor syndrome. Am J Hum Genet. 2012; 90(2): 308-313.

5. Le Goff C, Mahaut C, Bottani A, Doray B, Goldenberg A, Moncla A, et al. Not all Floating-Harbor syndrome cases are due to mutations in exon 34 of SRCAP. Hum Mutat. 2013; 34(1): 88-92.

6. Nikkel SM, Dauber A, de Munnik S, Connolly M, Hood RL, Caluseriu O, et al. The phenotype of Floating-Harbor syndrome: Clinical characterization of 52 individuals with mutations in exon 34 of SRCAP. Orphanet J Rare Dis. 2013; 8:63.

7. Kehrer M, Beckmann A, Wyduba J, Finckh U, Dufke A, Gaiser U, et al. Floating-Harbor syndrome: SRCAP mutations are not restricted to exon 34. Clin Genet 2014; 85(5): 498-499.

8. Seifert W, Meinecke P, Krüger G, Rossier E, Heinritz W, Wüsthof A. Expanded spectrum of exon 33 and 34 mutations in SRCAP and follow-up in patients with Floating-Harbor syndrome. BMC Med Genet. 2014; 15: 127.

9. Lacombe D, Patton M A, Elleau C, Battin J. FloatingHarbor syndrome: description of a further patient, review of the literature, and suggestion of autosomal dominant inheritance. Eur J Pediatr. 1995; 154(8): 658-661. 